Pacific Journal of Mathematics

ON ORTHOGONAL COMPLETION OF REDUCED RINGS 


\title{
ON ORTHOGONAL COMPLETION OF REDUCED RINGS
}

\author{
R. K. RAI
}

\begin{abstract}
It was proved by the author earlier that every orthogonal extension of a reduced ring $R$ is a subring of $Q(R)$, the maximal two sided ring of quotients of $R$ and the orthogonal completion of $R$, if it exists, is unique upto an isomorphism. Here, in Theorem 2, we prove that the orthogonal completion of $R$, if it exists, is a ring of right quotients $Q_{F}(R)$ of $R$ with respect to an idempotent filter $F$ of dense right ideals of $R$. Furthermore, it is shown in Proposition 5 that $Q_{F}(R)$ is an orthogonal extension of $R$ if and only if for every $q \in Q_{F}(R)$, there exists a maximal orthogonal subset $\left\{e_{i}: i \in I\right\}$ of idempotents of $Q(R)$ such that $q$ maps (by left multiplication) the right $R$-submodule of $Q(R)$ generated by $q^{-1} R \cup\left\{e_{i}\right.$ : $i \in I\}$ into $R$. Also an orthogonal extension $Q_{F}(R)$ is an orthogonal completion of $R$ if and only if for every $R$-submodule $M_{R}$ of $Q(R)_{R}$ generated by a maximal orthogonal subset of idempotents of $Q(R)$ and for every $f \in \operatorname{Hom}_{R}(M, R)$ there exists a $q \in Q_{F}(R)$ such that $f(m)=$ $q m$ for every $m \in M$ (Proposition 6). Thus we obtain a necessary and sufficient condition for a reduced ring to have an orthogonal completion without any reference to its idempotent which improves earlier known results derived by Burgess and Raphael. By examples we show that reduced rings without proper idempotents may also have an orthogonal completion.
\end{abstract}

Introduction. Abian [2] showed that the canonical order relation ' $\leq$ ' of Boolean rings can be defined for reduced rings $R$ (a ring with no nonzero nilpotent element) by writing $a \leq b$ if $a b=a^{2}$ and this order relation makes $R$ into a partially ordered multiplicative semigroup. Reduced rings under this relation ' $\leq$ ', were studied by Abian [1] and Chacron [5] to characterise the direct produce of integral domains, division rings and fields. Their studies involved the concepts of orthogonal completeness and orthogonal completion of reduced rings. These two concepts, on their own merit, were studied by Burgess, Raphael and Stephenson [3], [4], [11]. They proved that reduced rings which have enough idempotents ( $i$-dense) or satisfy certain chain conditions have an orthogonal completion. In this paper we shall provide a necessary and sufficient condition for a reduced ring to have an orthogonal completion.

In what follows, all rings referred to will have 1 , the identity element, and $R$ will always denote a reduced ring. In a reduced ring $R$, every 
idempotent is central and for every subset $X$ of $R$, right and left annihilators of $X$ in $R$ coincide. A subset $X$ of $R$ is called an orthogonal subset of $R$ if for every $x, y \in X, x \neq y$ implies $x y=0$. An element $a \in R$ is said to be an upper bound of an orthogonal subset $X$ of $R$ if $x a=x^{2}$ for every $x \in X$. An upper bound $a$ of $X$ is called a supremum of $X$ in $R$ if for every upper bound $b$ of $X$ in $R, a \leq b$. It is obvious from this definition that a supremum of an orthogonal subset of $R$, if it exists, is unique. We denote the supremum of an orthogonal subset $X$ in $R$ by $\sup _{R} X$. It can be easily proved that an upper bound $a$ of an orthogonal subset $X$ of $R$ is the supremum of $X$ in $R$ if and only if $\operatorname{ann}_{R} X=\operatorname{ann}_{R}(a)$. Also, for every orthogonal subset $X$ of $R$ and for every $r \in R, \sup _{R} r X=r\left(\sup _{R} X\right)$ provided $\sup _{R} X$ exists (see Raphael and Stephenson [11], page 347).

A reduced ring $R$ is said to be orthogonally complete if every orthogonal subset $X$ of $R$ has a supremum in $R$. A reduced ring $\hat{R} \supseteq R$ is an orthogonal extension of $R$ if every element of $\hat{R}$ is the supremum of an orthogonal subset of $R$. An orthogonal extension $\hat{R}$ of $R$ is said to be an orthogonal completion of $R$ if every orthogonal subset of $R$ has a supremum in $\hat{R}$. It follows easily from this that an orthogonal extension $\hat{R}$ of $R$ is an orthogonal completion of $R$ if and only if $\hat{R}$ is orthogonally complete. Obviously, every orthogonal extension of a reduced ring is a reduced ring.

A ring $S \supseteq R$ is said to be a ring of right quotients of $R$ if for every $s \in S, s^{-1} R=\{r \in R: s r \in R\}$ is a dense right ideal of $R$ and $s\left(s^{-1} R\right) \neq$ 0 . We denote the ring of right quotients of $R$ with respect to the idempotent filter of all dense right ideals of $R$ by $Q_{r}(R)$. Every ring of right quotients may be regarded as a subring of $Q_{r}(R)$ in the canonical way ([8], page 99).

Let $Q(R)=\left\{q \in Q_{r}(R): D q \subseteq R\right.$ for some dense left ideal $D$ of $\left.R\right\}$. Then obviously, $R \subseteq Q(R)$. It is proved by Wong and Johnson [14] that $Q(R)$ is a subring of $Q_{r}(R)$ and it is unique (up to isomorphism over $R$ ) maximal two sided ring of quotients of $R$. Also for every reduced ring $R$, $Q(R)$ is reduced (see Steinberg [12], page 466). It is proved in [10] (page 483) that every orthogonal subset $X$ of $R$ has a supremum in $Q(R)$ and since $Q(Q(R))=Q(R), Q(R)$ is orthogonally complete.

For every non-zero element $a$ of a reduced ring $R$ there exists an idempotent $0 \neq e \in Q(R)$ such that $\operatorname{ann}_{Q(R)}(a)=\operatorname{ann}_{Q(R)}(e)$ and $a e=$ $e a=a$. For a proof of this see Lambek [7], Theorem 6.6.

We denote the injective hull of a right $R$-module $M$ by $I(M)$.

LEMMA 1. Let $R$ be a ring, $S$ a proper subring of $Q_{r}(R)$ such that $R \subseteq S$ and $D$ a right ideal of $R$. Then $\operatorname{Hom}_{R}\left(R / D, I\left(Q_{r}(R) / S\right)\right)=0$ if and only 
if for every $x \in R$ and for every $q \in Q_{r}(R),(D: x) \subseteq(S: q)$ implies $q \in S$.

$$
\begin{aligned}
\text { Proof. } \operatorname{Hom}_{R}\left(R / D, I\left(Q_{r}(R) / S\right)\right)=0 \\
\Leftrightarrow \quad \text { for every } x+D \in R / D \text { and for every } 0 \neq q+S \in Q_{r}(R) / S \\
\quad \text { there exists an } r \in R \text { such that } x r \in D \text { and } q r \notin S \\
\Leftrightarrow \quad \text { for every } x \in R \text { and for every } q \in Q_{r}(R) \backslash S, \text { there } \\
\quad \text { exists an } r \in R \text { such that } r \in(D: x) \text { and } r \notin(S: q) \\
\Leftrightarrow \quad \text { for every } x \in R \text { and for every } q \in Q_{r}(R) \backslash S,(D: x) \nsubseteq \\
\quad(S: q) \\
\Leftrightarrow \quad \text { for every } x \in R \text { and for every } q \in Q_{r}(R),(D: x) \subseteq(S: q) \\
\quad \text { implies } q \in S .
\end{aligned}
$$

THEOREM 2. Let $R$ be a reduced ring which admits an orthogonal completion $\hat{R}$. Then there exists an idempotent filter $\mathbf{F}$ of dense right ideals of $R$ such that $\hat{R}=Q_{F}(R)$.

Proof. If $\hat{R}=Q_{r}(R)$, then $\hat{R}=Q_{F}(R)$ where $\mathbf{F}$ is the idempotent filter of all dense right ideal of $R$. Hence assume that $\hat{R} \subset Q_{r}(R)$ and let F denote the collection of all those dense right ideals of $R$ for which $\operatorname{Hom}_{R}\left(R / D, I\left(Q_{r}(R) / \hat{R}\right)\right)=0$. Then $\mathbf{F}$ is an idempotent filter of dense right ideals of $R$ corresponding to the torsion theory cogenerated by $I\left(Q_{r}(R) / \hat{R}\right)$. Now consider $Q_{F}(R)$, which is a subring of $Q_{r}(R)$. Let $q \in Q_{F}(R)$. Then there exists a $D \in \mathbf{F}$ such that $q D \subseteq R$. Hence $D=$ $(D: 1) \subseteq(\hat{R}: q)$, which by Lemma 1 implies that $q \in \hat{R}$. Thus $Q_{F}(R) \subseteq$ $\hat{R}$.

On the other hand, let $q \in \hat{R}$ and suppose $0 \neq f \in$ $\operatorname{Hom}_{R}\left(R / q^{-1} R, I\left(Q_{r}(R) / \hat{R}\right)\right)$. Let $0 \neq p+\hat{R} \in f\left(R / q^{-1} R\right) \cap Q_{r}(R) / \hat{R}$ and $a \in R$ be such that $f\left(a+q^{-1} R\right)=p+\hat{R}$. Then for every $r \in$ $\left(q^{-1} R: a\right)=(R: q a), f\left(a+q^{-1} R\right) r=0$. Hence $p(R: q a) \subseteq \hat{R}$.

Since $q \in \hat{R}$ and $a \in R, q a, q a+1 \in \hat{R}$. Also, $(R: q a)=(R: q a+$ 1). Hence there exist orthogonal subsets $X=\left\{x_{i}: i \in I\right\}, Y=\left\{y_{j}: j \in J\right\}$ of $(R: q a)$ such that $q a=\sup _{\hat{R}} X$ and $q a+1=\sup _{\hat{R}} X$. Since $p(R: q a)$ $\subseteq \hat{R}, p X, p Y \subseteq \hat{R}$. Hence for every $z \in X$ and $r \in R$,

$$
\begin{aligned}
\left.\sup _{\hat{R}} p X\right)(r z) & =\sup _{\hat{R}}[(p X)(r z)] & & (\text { Cor. 1.2, [11]) } \\
& =\sup _{\hat{R}} p[X(r z)] & & \text { (associativity) } \\
& =p(z r z) & & \text { (orthogonality of } X) .
\end{aligned}
$$


Also, since $q a=\sup _{\hat{R}} X, z(q a)=(q a) z=z^{2}$. Hence $[(q a) r z-z r z]^{2}=0$ and since $\hat{R}$ is a reduced ring, this implies that $(q a) r z=z r z$ for every $z \in X$ and $r \in R$. Now consider the dense ideal $D=\left\{\sum a_{i} x_{i} b_{i}+r: a_{i}, b_{i}\right.$ $\in R, x_{i} \in X$ and $\left.r \in \operatorname{ann}_{R} X\right\}$. For every $\sum a_{i} x_{i} b_{i}+r \in D$

$$
\begin{aligned}
\left(\sup _{\hat{R}} p X\right)\left(\sum a_{i} x_{i} b_{i}+r\right) & =\sum\left(\sup _{\hat{R}} p X\right) a_{i} x_{i} b_{i} \quad \text { (Prop. 1.1, [11]) } \\
& =\sum p\left(x_{i} a_{i} x_{i} b_{i}\right) \\
& =p\left(\sum(q a) a_{i} x_{i} b_{i}\right) \\
& =p(q a)\left(\sum a_{i} x_{i} b_{i}+r\right)
\end{aligned}
$$

Hence $\left(p q a-\sup _{\hat{R}} p X\right) D=0$ proving that $\sup _{\hat{R}} p X=p q a$. Similarly, $p(q a+1)=\sup _{\hat{R}} p Y$. But this implies that $p \in \hat{R}$ which contradicts the fact that $p+\hat{R}$ is a nonzero element of $Q_{r}(R) / \hat{R}$. Hence $\operatorname{Hom}_{R}\left(R / q^{-1} R, I\left(Q_{r}(R) / \hat{R}\right)\right)=0$ proving that $q^{-1} R \in \mathbf{F}$. Therefore $q \in$ $Q_{F}(R)$ and hence $\hat{R}=Q_{F}(R)$ as was required to be proved.

As an immediate consequence of this theorem we have the following result.

Proposition 3. Let $R$ be a reduced indecomposable ring which has an orthogonal completion $R$. Let $\mathbf{F}$ be an idempotent filter of dense right ideals of $R$ such that $\hat{R}=Q_{F}(R)$. Then no dense right ideal $D \in \mathbf{F}$ can be expressed as a nontrivial direct sum of two right ideals.

Proof. If $D=D_{1} \oplus D_{2}$, then $e \in Q_{F}(R)$ which is defined by $e\left(d_{1}+d_{2}\right)=d_{1}$ for every $d_{1} \in D_{1}, d_{2} \in D_{2}$ is an idempotent of $\hat{R}=$ $Q_{F}(R)$. If $D_{1} \neq 0 \neq D_{2}$, then $e$ is a proper idempotent of $\hat{R}$. Since $\hat{R}$ is an orthogonal completion of $R$ and $e \in \hat{R}$, there exists an orthogonal set $\left\{e_{I}\right.$ : $i \in R\} \subseteq R$ such that $e=\sup _{\hat{R}} e_{i}$. But then each $e_{i}$ should be a proper idempotent of $R$ which is a contradiction since $R$ is indecomposable.

COROLlaRY 4. $C[0,1]$, the ring of continuous real values function on the interval $[0,1]$ has no orthogonal completion.

Proof. Consider the orthogonal subset $\left\{f_{n}: n \in N\right\} \subseteq C[0,1]$, where for a typical $n \in N, f_{n}$ is given by the following. 


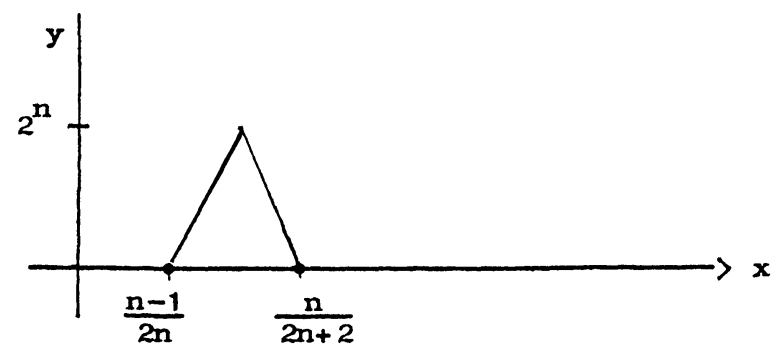

Then $\sup f_{n}$ is not continuous at $1 / 2$. Hence $C[0,1]$ is not orthogonally complete. Now suppose $C[0,1]$ has an orthogonal completion $\hat{R}$. Then by Theorem 2 there exists an idempotent filter $\mathbf{F}$ of dense ideals of $C[0,1]$ such that $\hat{R}=Q_{F}(C[0,1])$. Let $f \in Q(C[0,1])$ be the supremum of $\left\{f_{n}\right.$ : $n \in N\}$ and $M=\{g \in C[0,1]: g(1 / 2)=0\}$. The $f^{-1}(C[0,1])=\{g \in$ $C[0,1]: f g \in C[0,1]\} \subseteq M$. Hence $M \in \mathbf{F}$. Since $C[0,1]$ has no proper idempotents and $M$ can be expressed as a proper direct sum of ideals of $C[0,1]$, by Proposition 3 this is a contradiction. Hence $C[0,1]$ has no orthogonal completion.

Burgess and Raphael [3] proved this result using the properties of continuous real valued functions over a closed interval.

Theorem 2 provides us a necessary condition for a reduced ring $\hat{R}$ to be an orthogonal completion of $R$. But it fails to provide a sufficient condition for a reduced ring $R$ to have an orthogonal completion because for many subrings $S$ of $Q_{r}(R)$ there exist idempotent filters $\mathbf{F}$ such that $S=Q_{F}(R)$. The following proposition characterises the nature of idempotent filters which give rise to an orthogonal extension of $R$.

In what follows, we shall write $\sup X$ for $\sup _{Q(R)} X$. Also, we would like to remind our reader that for an orthogonal subset $X$ of $R$, Burgess and Raphael ([3], Lemma 11) proved that $\sup _{R} X=\sup X$ whenever $\sup _{R} X$ exists.

Proposition 5. Let $R$ be a reduced ring and $\mathbf{F}$ an idempotent filter of dense right ideals of $R$. Then $Q_{F}(R)$ is an orthogonal extension of $R$ if and only if for every $q \in Q_{F}(R)$, there exists a maximal orthogonal subset $\left\{e_{i}\right.$ : $i \in I\}$ of idempotents of $Q(R)$ such that $q$ maps (by left multiplication) the right $R$-submodule of $Q(R)$ generated by $q^{-1} R \cup\left\{e_{i}: i \in I\right\}$ into $R$.

Proof. Suppose $Q_{F}(R)$ is an orthogonal extension of $R$. Then $Q_{F}(R)$ $\subseteq Q(R)$. Let $a \in Q_{F}(R)$. Then there exists an orthogonal subset $\left\{a_{i}\right.$ : $\left.i \in I^{\prime}\right\}$ of $R$ such that $a=\sup \left\{a_{i}: i \in I^{\prime}\right\}$. Let $E=\left\{e_{i}: i \in I^{\prime}\right\}$ be the 
orthogonal subset of central idempotents of $Q(R)$ such that $a_{i}, e_{i}=a_{i}$ and $\operatorname{ann}_{Q(R)}\left(a_{i}\right)=\operatorname{ann}_{Q(R)}\left(e_{i}\right)$ for every $i \in I^{\prime}$ (see [7], Theorem 6.6).

Embed $E$ in a maximal orthogonal subset $\left\{e_{i}: i \in I\right\}$ of idempotents of $Q(R)$. Then, since

$$
\operatorname{ann}_{Q(R)}\left\{a_{i}: i \in I^{\prime}\right\}=\operatorname{ann}_{Q(R)}(a)=\operatorname{ann}_{Q(R)}\left\{e_{i}: i \in I^{\prime}\right\},
$$

$a e_{i}=0$ for every $i \in I \backslash I^{\prime}$. Also, since $a$ is the supremum of $\left\{a_{i}: i \in I^{\prime}\right\}$, $a_{i}=a_{i} e_{i} \leq a e_{i}$ for every $i \in I^{\prime}$. But, since

$$
\operatorname{ann}_{Q(R)}\left(e_{i}\right) \subseteq \operatorname{ann}_{Q(R)}\left(a e_{i}\right) \subseteq \operatorname{ann}_{Q(R)}\left(a_{i}\right),
$$

we have $a_{i}=a e_{i}$, proving that $a e_{i} \in R$ for every $i \in I$.

Now if we let $M$ be the right $R$-submodule of $Q(R)$ generated by $q^{-1} R \cup\left\{e_{i}: i \in I\right\}$ then obviously the left multiplication by $q$ determines a homomorphism from $M$ into $R$.

Conversely, suppose for every $q \in Q_{F}(R)$ there exists a maximal orthogonal subset $\left\{e_{i}: i \in I\right\}$ of idempotents of $Q(R)$ such that $q$ maps (by left multiplication) the right $R$-submodule of $Q(R)$ generated by $q^{-1} R \cup\left\{e_{i}: i \in I\right\}$ into $R$. Consider the ideal $D=\left\{\sum e_{i} R\right\} \cap R$. Since $\left\{e_{i}: i \in I\right\}$ is a maximal orthogonal subset of $Q(R), \operatorname{ann}_{R} D=0$. Hence $D$ is a dense right and left ideal of $R$. Since $e_{i}$ 's are central in $Q(R)$, $D q \subseteq R$ and therefore $q \in Q(R)$. It follows from this that $Q_{F}(R) \subseteq Q(R)$.

Now, since $\left\{e_{i}: i \in I\right\}$ is a maximal orthogonal subset of $Q(R), q$ is the supremum of the orthogonal subset $\left\{q e_{i}: i \in I\right\}$ of $R$. Hence $Q_{F}(R)$ is an orthogonal extension of $R$. This completes the proof.

The following proposition gives us a necessary and sufficient condition for an orthogonal extension to be an orthogonal completion.

Proposition 6. Let $\mathbf{F}$ be an idempotent filter of dense right ideals of $R$ such that $Q_{F}(R)$ is an orthogonal extension of $R$. Then $Q_{F}(R)$ is an orthogonal completion of $R$ if and only if for every $R$-submodule $M_{R}$ of $Q(R)_{R}$ generated by a maximal orthogonal subset of idempotents of $Q(R)$ and for every $f \in \operatorname{Hom}_{R}(M, R)$ there exists a $q \in Q_{F}(R)$ such that $f(m)=$ qm for every $m \in M$.

Proof. Since $Q_{F}(R)$ is an orthogonal extension of $R . Q_{F}(R)$ is a subring of $Q(R)$. Suppose $Q_{F}(R)$ is an orthogonal completion of $R, M_{R}$ is an $R$-submodule of $Q(R)$ generated by a maximal orthogonal subset $\left\{e_{i}\right.$ : $i \in I\}$ of idempotents of $Q(R)$ and $f \in \operatorname{Hom}_{R}(M, R)$. Then (as in the proof of Proposition 5) the ideal $D=\left(\sum_{i} e_{i} R\right) \cap R=M \cap R$ of $R$ is a 
dense right and left ideal of $R$. Since $f \mid D \in \operatorname{Hom}_{R}\left(D_{R}, R_{R}\right)$, we can find a $q \in Q_{r}(R)$ such that $f(d)=q d$ for every $d \in D$. Let $i \in I$. Then since $D$ is a dense right ideal of $R$ and $e_{i} \in Q_{r}(R), e_{t}^{-1} D$ is $a$ dense right ideals of $R$. Now, $q e_{l}-f\left(e_{i}\right) \in Q_{r}(R)$ and for every $r \in e_{i}^{-1} D$,

$$
\begin{aligned}
\left(q e_{i}-f\left(e_{\imath}\right)\right) r & =q e_{i} r-f\left(e_{l}\right) r \\
& =f\left(e_{\imath} r\right)-f\left(e_{\imath}\right) r \\
& =f\left(e_{i}\right) r-f\left(e_{i}\right) r \\
& =0 .
\end{aligned}
$$

Since $e_{l}^{-1} D$ is a dense right ideal of $R$, it follows from this that $q e_{i}=f\left(e_{l}\right)$ for every $i \in I$. Hence $f(m)=q m$ for every $m \in M$. Further, for every $i \in I$ and $d \in D$,

$$
\begin{aligned}
\left(q e_{l}-e_{i} q\right) d & =q\left(e_{l} d\right)-e_{l}(q d)=f\left(e_{l} d\right)-e_{i} f(d) \\
& =q\left(d e_{l}\right)-(q d) e_{\imath}=0 .
\end{aligned}
$$

(Here we used the fact that $e_{i}$ 's are central in $Q(R)$ and that $q d \in R$.) Since $D$ is a dense right ideal of $R$, it follows from this that $q e_{i}=e_{i} q$ for every $i \in I$. Therefore, $D q=\left(\left(\sum_{i} e_{i} R\right) \cap R\right) q \subseteq R$. Thus $q \in Q(R)$ and hence $\sup \left\{q e_{l}: i \in I\right\}=q \cdot \sup \left\{e_{l}: i \in I\right\}=q \cdot 1=q$. Since $\left\{q e_{i}: i \in I\right\}$ $\subseteq R \subseteq Q_{F}(R)$ and $Q_{F}(R)$ is orthogonally complete, this implies that $q \in Q_{F}(R)$, as was required.

Now we prove the converse. Let $\left\{a_{i}: i \in I^{\prime}\right\} \subseteq R$ be an orthogonal subset and $\left\{e_{i}: i \in I^{\prime}\right\}$ be the orthogonal subset of idempotents of $Q(R)$ such that $a_{i} e_{l}=e_{i} a_{i}=a_{i}$ and $\operatorname{ann}_{Q(R)}\left(a_{\imath}\right)=\operatorname{ann}_{Q(R)}\left(e_{l}\right)$ for every $i \in I^{\prime}$. Let $\left\{e_{i}: i \in I\right\}$ be a maximal orthogonal subset of idempotents of $Q(R)$ containing $\left\{e_{l}: i \in I^{\prime}\right\}$ and $M=\sum_{i \in I} e_{l} R$. Define $f: M \rightarrow R$ by

$$
\begin{aligned}
f\left(e_{i}\right) & =a_{i} & & \text { if } i \in I^{\prime} \\
& =0 & & \text { otherwise }
\end{aligned}
$$

and extend $f$ by linearity over sums. Then $f \in \operatorname{Hom}_{R}(M, R)$. Hence by our assumption there exists an $a \in Q_{F}(R)$ such that $f(m)=$ am for every $m \in M$. Now

$$
a a_{\imath}=a\left(a_{\imath} e_{\imath}\right)=a\left(e_{i} a_{\imath}\right)=f\left(e_{i}\right) a_{i}=a_{t}^{2}
$$

and hence $a_{l} \leq a$ for every $i \in I^{\prime}$. Also, if $x \in \operatorname{ann}_{Q(R)}\left\{a_{i}: i \in I^{\prime}\right\}$ then, since

$$
\left(a e_{i}\right) x=f\left(e_{i}\right) x
$$


$\left(a e_{i}\right) x=0$ for every $i \in I$. Hence,

$$
a x=a .1 . x=a\left(\sup \left\{e_{i}: i \in I\right\}\right) x=\sup \left\{\left(a e_{i}\right) x: i \in I\right\}=0 .
$$

Thus $\operatorname{ann}_{Q(R)}\left\{a_{i}: i \in I^{\prime}\right\} \subseteq \operatorname{ann}_{Q(R)}(a)$. Since $a$ is an upper bound of $\left\{a_{i}: i \in I^{\prime}\right\}, \operatorname{ann}_{Q(R)}(a) \subseteq \operatorname{ann}_{Q(R)}\left\{a_{i}: i \in I^{\prime}\right\}$. Hence $a$ is the supreinum of $\left\{a_{i}: i \in I^{\prime}\right\}$. Since, $Q_{F}(R) \subseteq Q(R)$ and $a \in Q_{F}(R), a=$ $\sup _{Q_{F}(R)}\left\{a_{i}: i \in I^{\prime}\right\}$. Thus we see that every orthogonal subset of $R$ has a supremum in $Q_{F}(R)$. Since $Q_{F}(R)$ is an orthogonal extension of $R$, it follows that $Q_{F}(R)$ is the orthogonal completion of $R$. This completes the proof.

Combining Propositions 5 and 6 we get the following result.

THEOREM 7. A reduced ring $R$ has an orthogonal completion if and only if it has an idempotent filter $\mathbf{F}$ of dense right ideals of $R$ such that (i) for every $q \in Q_{F}(R)$ there exists a maximal orthogonal subset $\left\{e_{i}: i \in I\right\}$ of idempotents of $Q(R)$ such that $q$ maps (by left multiplication), the right $R$-submodule $M$ of $Q(R)$ generated by $q^{-1} R \cup\left\{e_{i}: i \in I\right\}$ into $R$ and

(ii) for every $R$-submodule $M$ of $Q(R)$ generated by maximal orthogonal subset of idempotents of $Q(R)$ and for every $f \in \operatorname{Hom}_{R}(M, R)$, there exists a $q \in Q_{F}(R)$ such that $f(m)=$ qm for every $m \in M$.

Thus a reduced ring $R$ has an orthogonal completion if and only if it has an idempotent filter $\mathbf{F}$ of dense right ideals of $R$ such that $Q_{F}(R)$ consists of exactly those elements of $Q(R)$ for which there exists a maximal orthogonal subset $\left\{e_{i}: i \in I\right\}$ of idempotents of $Q(R)$ such that $q e_{i} \in R$ for every $i \in I$. Also, it follows from this that a reduced ring $R$ is orthogonally complete if and only if it contains all those elements of $Q(R)$ for which there exists a maximal orthogonal subset $\left\{e_{i}: i \in I\right\}$ of idempotents of $Q(R)$ such that $q e_{i} \in R$ for every $i \in I$.

As an application of Theorem 7, we prove the following result which was established in [4] for commutative rings.

COROLlARY 8. Every reduced $i$-dense ring has an orthogonal completion.

Proof. Let $\mathbf{F}$ be the idempotent filter of all those dense right deals of $R$ which contain a maximal orthogonal subset of idempotents of $R$ and consider $Q_{F}(R)$. Since all members of $\mathbf{F}$ contain a maximal orthogonal subset of idempotents of $R, Q_{F}(R)$ is an orthogonal extension of $R$. Now let $M_{R}$ be any $R$-submodule of $Q(R)_{R}$ generated by a maximal orthogonal 
subset of idempotents of $Q(R)$ and $f \in \operatorname{Hom}_{R}(M, R)$. Then, since each idempotent of $Q(R)$ is the supremum of an orthogonal subset of idempotents of $R$, it follows that $M_{R}$ contains a maximal orthogonal subset of idempotents of $R$ and hence a member $D$ of $\mathbf{F}$. Therefore, there exists a $q \in Q_{F}(R)$ such that $f(d)=q d$ for every $d \in D$.

Now let $m$ be an arbitrary element of $M$. Then, since $M \subseteq Q(R)$, $m^{-1} D=\{r \in R: m r \in D\}$ is a dense right ideal of $R$ and for every $d^{\prime} \in m^{-1} D, m d^{\prime} \in D$ and hence

$$
(f(m)-q m) d^{\prime}=f(m) d^{\prime}-(q m) d^{\prime}=f\left(m d^{\prime}\right)-q\left(m d^{\prime}\right)=0 .
$$

Thus $f(m)=q m$ for every $m \in M$ and hence by Theorem $7 Q_{F}(R)$ is the orthogonal completion of $R$. This completes the proof.

It follows from this result that every reduced Baer ring has an orthogonal completion (see [5], Theorems 18).

EXAMPLE 9. For every $n \geq 1$, let $R_{n}=k[x, y, z]$ be a polynomial ring in commuting indeterminates over a field $k$ such that $y z=0$. Let $R$ be the subring of $\prod_{n=1}^{\infty} R_{i}$ generated by $\bigoplus_{n=1}^{\infty} R_{n}$ and $1 \in \prod_{n=1}^{\infty} R_{n}$. Let $D=$ $\bigoplus_{n=1}^{\infty} R_{n}$ and $\mathbf{F}$ be the idempotent filter of ideals of $R$ which contain $D$. Since $D$ contains a maximal orthogonal subset of idempotents of $R$ and hence of $Q(R)$, it follows from Theorem 7 that $Q_{F}(R)$ is an orthogonal extension of $R$. Also, since every $R$-submodule $M$ of $Q(R)$ generated by a maximal orthogonal subset $\left\{f_{i}: i \in I\right\}$ of idempotents of $Q(R)$ contains $\left\{e_{n}: n \in N\right\}$ (where $e_{n}$ is that element of $R \subseteq \prod_{i=1}^{\infty} R_{i}$ whose $n$th coordinate is 1 and all other coordinates are 0 ), it follows that $D \subseteq M$. Hence if $g \in \operatorname{Hom}_{R}(M, R)$ then $g \mid D \in \operatorname{Hom}_{R}(D, R)$. Let $q \in Q_{F}(R)$ be such that $g(d)=q d$ for every $d \in D$.

Now, let $m$ be an arbitrary element of $M$. Then as in the proof of Corollary 8 , it can be proved that $g(m)=q m$ for every $m \in M$. Hence by Theorem 7, $Q_{F}(R)$ is the orthogonal completion of $R$. It can be easily verified that $Q_{F}(R)=\prod_{i=1}^{\infty} R_{i}$. It is also interesting to observe that $R$ is not $i$-dense.

Theorem 7 produces conclusive results when applied to reduced rings without proper idempotents as well. This is done in the next example.

EXAMPLE 10. Let $S$ denote the ring $\prod_{n=1}^{\infty} k_{n}[x]$ where $k_{n}=\mathbf{Z} /(2)$ for every $n \in N$ and let $M=\left(\mathbf{x}^{2}+\mathbf{x}\right) S$ where $\mathbf{x}$ is the element of $S$ all of 
whose co-ordinates are $x$. Let $R$ be the subring of $S$ generated by $M \cup\{\mathbf{1}\} \cup\left\{a_{n}: n \in \mathbf{N}\right\} \cup\left\{b_{n}: n \in \mathbf{N}\right\}$ where

$$
\begin{aligned}
& a_{1}=(x+1, x+1,0,0,0,0,0, \ldots \ldots \ldots \ldots \ldots \ldots) \text {, } \\
& a_{2}=(0,0, x+1, x+1,0,0, \ldots \ldots \ldots \ldots \ldots \ldots \ldots) \text {, } \\
& a_{3}=(0,0,0,0, x+1, x+1,0,0, \ldots \ldots \ldots \ldots \ldots) \text {, } \\
& b_{1}=(x, 0,0,0,0,0,0, \ldots \ldots \ldots \ldots \ldots \ldots \ldots \ldots) \text {, } \\
& b_{2}=(0, x, x, 0,0,0,0, \ldots \ldots \ldots \ldots \ldots \ldots \ldots \ldots \ldots) \text {, } \\
& b_{3}=(0,0,0, x, x, 0,0, \ldots \ldots \ldots \ldots \ldots \ldots \ldots \ldots \ldots \ldots \ldots \ldots \ldots \ldots \ldots .
\end{aligned}
$$

Every power of $a_{n}$ (resp. $b_{n}$ ) can be expressed as $a_{n}+m$ (resp. $b_{n}+m$ ) where $m \in M$. Also, $a_{n} b_{m} \in M, a_{n} M \subseteq M$ and $b_{n} M \subseteq M$. Hence every element of $R$ can be expressed in the form

$$
\sum \alpha_{i} a_{i}+\sum \beta_{j} b_{j}+m+\mathbf{n}
$$

where $\alpha_{i}, \beta_{j} \in \mathbf{Z} /(2)$ for every $i, j, m \in M$ and $\mathbf{n}=\mathbf{0}$ or $\mathbf{1}$. It can be easily verified that $R$ has no proper idempotent.

Let $D$ be the ideal of $R$ generated by $\left\{a_{n}: n \in \mathbf{N}\right\},\left\{b_{n}: n \in \mathbf{N}\right\}$ and let $\mathbf{F}$ be the idempotent filter of all those ideals of $R$ which contain $D$. Since $\operatorname{ann}_{R} D=0, \mathbf{F}$ is an idempotent filter of dense ideals of $R$. Let $f$ : $D \rightarrow R$ be an $R$ homomorphism. Then since (taking $a_{0}=0$ )

$$
\begin{aligned}
& a_{i}=\left(b_{i}+a_{i}+b_{i+1}\right) a_{i}, \\
& b_{i}=\left(a_{i-1}+b_{i}+a_{i}\right) b_{i},
\end{aligned}
$$

it follows that

$$
f\left(a_{i}\right)=\left[f\left(b_{i}\right)+f\left(a_{i}\right)+f\left(b_{i+1}\right)\right] a_{i}
$$

and

$$
f\left(b_{i}\right)=\left[f\left(a_{i-1}\right)+f\left(b_{i}\right)+f\left(a_{i}\right)\right] b_{i} .
$$

Let $q=\sup _{i}{ }_{Q(R)} f\left(a_{i}\right)+\sup _{j(R)} f\left(b_{j}\right)$. Then, since every element of $D$ is of the form of

$$
d=\sum \alpha_{i} a_{i}+\sum \beta_{j} b_{j}+m
$$

where $\alpha_{i}, \beta_{j} \in \mathbf{Z} /(2)$ and $m=\Sigma a_{k} r_{k}$ (or $\Sigma b_{k} s_{k}$ ) is a finite sum with $r_{k}$ 's (or $s_{k}$ 's) in $R$. Hence it follows that $f(d)=q d$ for every $d \in D$. We show 
that either $q \in R$ or there exists a proper maximal orthogonal subset $E=\left\{e_{i}: i \in I\right\}$ of idempotents of $Q \max (R)$ such that $q E \subseteq R$.

Since $f\left(b_{i}\right)+f\left(a_{i}\right)+f\left(b_{i+1}\right) \in R$, it is of the form $\sum \alpha_{i} a_{i}+\sum \beta_{j} b_{j}+$ $m+\mathbf{n}$ and it follows from this that $f\left(a_{i}\right)=\alpha_{1}^{\prime} a_{i}+m_{i}$ where $m_{i} \in M$ is such that $m_{l} a_{j}=0$ for every $i \neq j$ and $\alpha_{i}^{\prime} \in \mathbf{Z} /(2)$. Hence, by Lemma 5 of [10],

$$
\begin{aligned}
\sup _{i} f\left(a_{i}\right) & =\sup _{i}\left(\alpha_{i}^{\prime} a_{i}+m_{i}\right)=\sup _{i} \alpha_{i}^{\prime} a_{i}+\sup _{i} m_{i} \\
& =\sup \alpha_{i}^{\prime} a_{i}+m \quad \text { where } m \in M .
\end{aligned}
$$

Similarly,

$$
\begin{aligned}
\sup _{j} f\left(b_{j}\right) & =\sup _{j}\left(\beta_{j}^{\prime} b_{j}+m_{j}^{\prime}\right)=\sup _{j} \beta_{j}^{\prime} b_{j}+\sup _{j} m_{j}^{\prime} \\
& =\sup _{j} \beta_{j}^{\prime} b_{j}+m^{\prime} \quad \text { where } m^{\prime} \in M .
\end{aligned}
$$

Hence,

$$
q=\sup _{i} \alpha_{i}^{\prime} a_{i}+\sup \beta_{j}^{\prime} b_{j}+\left(m+m^{\prime}\right)
$$

where $\alpha_{i}^{\prime}, \beta_{j}^{\prime} \in \mathbf{Z} /(2)$ and $m+m^{\prime} \in M$. Thus $q=r+m^{\prime \prime}$ where $r=$ $\sup _{i} \alpha_{i}^{\prime} a_{i}+\sup _{j} \beta_{j}^{\prime} b_{j}$ and $m^{\prime \prime}=m+m^{\prime}$.

It follows from these discussions that $Q_{F}(R)$ is the subring of $S$ obtained by adjoining elements of the form of $\sup \alpha_{i} a_{i}+\sup \beta_{j} b_{j}$ to $R$ where $\alpha_{i}, \beta_{j} \in \mathbf{Z} /(2)$ for all $i, j$. Now using Theorem 7, it can be verified that $Q_{F}(R)$ is the orthogonal completion of $R$.

This example shows that the existence of proper idempotents in $R$ is not a necessary condition for $R$ to have an orthogonal completion.

\section{REFERENCES}

[1] A. Abian, Direct product decomposition of commutative semisimple rings, Proc. Amer. Math. Soc., 24 (1970), 502-507.

[2] Order in a special class of rings and a structure theorem, Proc. Amer. Math. Soc., 52 (1975), 45-49.

[3] W. D. Burgess and R. Raphael, Abian's order relation and orthogonal completions for reduced rings, Pacific J. Math., 54 (1974), 55-64.

[4] Complete and orthogonally complete rings, Canad. J. Math., 27 (1975), 884-892.

[5] M. Chacron, Direct product of division rings and a paper of Abian, Proc. Amer. Math. Soc., 24 (1970), 502-507.

[6] W. H. Cornish and P. N. Stewart, Weakly regular algebras, Boolean orthogonalities and direct product of integral domains, Canad. J. Math., 28 (1976), 148-153. 
[7] J. Lambek, On structure of semisimple rings and their rings of quotients, Canad. J. Math., 13 (1961), 392-417.

[8] __ Lectures on Rings and Modules, Chelsea publ. Co., New York, N. Y., (1976).

[9] - Torsion theories. Additive Semantics and Rings of Quotients, Lecture notes in Math., Springer-Verlag, 177 (1971).

[10] R. K. Rai, Orthogonal completion of reduced rings with respect to Abian Order, Canad. Math. Bull., 23 (1980), 477-489.

[11] R. Raphael and W. Stephenson, Orthogonally complete rings, Canad. Math. Bull., 20 (1977), 347-351.

[12] S. A. Steinberg, Rings of quotients of rings without nilpotent elements, Pacific J. Math., 49 (1973), 493-506.

[13] B. Stenstrom, Rings of Quotients, Springer-Verlag, New York, N. Y., (1975).

[14] E. T. Wong and R. E. Johnson, Self injective rings, Canad. Math. Bull., 2 (1959), 167-173.

Received May 8, 1980 and in revised form May 20, 1982.

DALHOUSIE UNIVERSITY

HalifaX, N. S. Canada B3H $4 \mathrm{H} 8$

Current address: Mehta Research Institute of Mathematics and Mathematical Physics 26, Dilkusha, New Katra

Allahabad-211 002

U. P., India 


\title{
PACIFIC JOURNAL OF MATHEMATICS EDITORS
}

\author{
Donald BabBitT (Managing Editor) \\ University of California \\ Los Angeles, CA 90024 \\ Hugo Rossi \\ University of Utah \\ Salt Lake City, UT 84112 \\ C. C. Moore and Arthur Ogus \\ University of California \\ Berkeley, CA 94720
}

\author{
J. DugundiI \\ Department of Mathematics \\ University of Southern California \\ Los Angeles, CA 90089-1113 \\ R. FINN and H. SAMELSON \\ Stanford University \\ Stanford, CA 94305
}

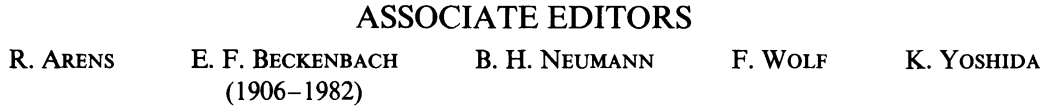

\section{SUPPORTING INSTITUTIONS}

UNIVERSITY OF ARIZONA
UNIVERSITY OF BRITISH COLUMBIA
CALIFORNIA INSTITUTE OF TECHNOLOGY
UNIVERSITY OF CALIFORNIA
MONTANA STATE UNIVERSITY
UNIVERSITY OF NEVADA, RENO
NEW MEXICO STATE UNIVERSITY
OREGON STATE UNIVERSITY

\author{
UNIVERSITY OF OREGON \\ UNIVERSITY OF SOUTHERN CALIFORNIA \\ STANFORD UNIVERSITY \\ UNIVERSITY OF HAWAII \\ UNIVERSITY OF TOKYO \\ UNIVERSITY OF UTAH \\ WASHINGTON STATE UNIVERSITY \\ UNIVERSITY OF WASHINGTON
}

The Supporting Institutions listed above contribute to the cost of publication of this Journal, but they are not owners or publishers and have no responsibility for its content or policies.

Mathematical papers intended for publication in the Pacific Journal of Mathematics should be in typed form or offset-reproduced (not dittoed), double spaced with large margins. Please do not use built up fractions in the text of the manuscript. However, you may use them in the displayed equations. Underline Greek letters in red, German in green, and script in blue. The first paragraph must be capable of being used separately as a synopsis of the entire paper. In particular it should contain no bibliographic references. Please propose a heading for the odd numbered pages of less than 35 characters. Manuscripts, in triplicate, may be sent to any one of the editors. Please classify according to the scheme of Math. Reviews, Index to Vol. 39. Supply name and address of author to whom proofs should be sent. All other communications should be addressed to the managing editor, or Elaine Barth, University of California, Los Angeles, California 90024.

There are page-charges associated with articles appearing in the Pacific Journal of Mathematics. These charges are expected to be paid by the author's University, Government Agency or Company. If the author or authors do not have access to such Institutional support these charges are waived. Single authors will receive 50 free reprints; joint authors will receive a total of 100 free reprints. Additional copies may be obtained at cost in multiples of 50 .

The Pacific Journal of Mathematics is issued monthly as of January 1966. Regular subscription rate: $\$ 132.00$ a year (6 Vol., 12 issues). Special rate: $\$ 66.00$ a year to individual members of supporting institutions.

Subscriptions, orders for numbers issued in the last three calendar years, and changes of address should be sent to Pacific Journal of Mathematics, P.O. Box 969, Carmel Valley, CA 93924, U.S.A. Old back numbers obtainable from Kraus Periodicals Co., Route 100, Millwood, NY 10546.

The Pacific Journal of Mathematics ISSN 0030-8730 is published monthly by the Pacific Journal of Mathematics at P.O. Box 969, Carmel Valley, CA 93924. Application to mail at Second-class postage rates is pending at Carmel Valley, California, and additional mailing offices. Postmaster: Send address changes to Pacific Journal of Mathematics, P. O. Box 969, Carmel Valley, CA 93924.

PUBLISHED BY PACIFIC JOURNAL OF MATHEMATICS, A NON-PROFIT CORPORATION

Copyright $(1983$ by Pacific Journal of Mathematics 


\section{Pacific Journal of Mathematics}

Vol. 108, No. $2 \quad$ April, 1983

Enrique Atencia and Francisco Javier Martin-Reyes, The maximal ergodic Hilbert transform with weights $\ldots \ldots \ldots \ldots \ldots \ldots \ldots \ldots \ldots . \ldots 257$

Bruce Blackadar, The regular representation of local affine motion

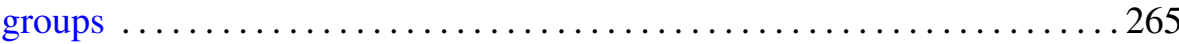

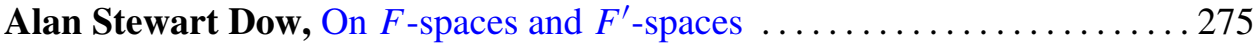

Yoshifumi Kato, On the vector fields on an algebraic homogeneous space . . 285

Dmitry Khavinson, Factorization theorems for different classes of analytic functions in multiply connected domains $\ldots \ldots \ldots \ldots \ldots \ldots \ldots \ldots 295$

Wei-Eihn Kuan, A note on primary powers of a prime ideal . . . . . . . . 319

Benjamin Michael Mann and Edward Yarnell Miller, Characteristic classes for spherical fibrations with fibre-preserving free group

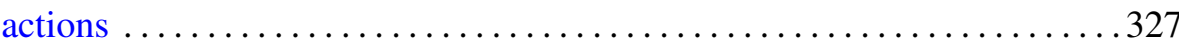

Steven Alan Pax, Appropriate cross-sectionally simple four-cells are flat . . . 379 R. K. Rai, On orthogonal completion of reduced rings ................ 385

V. Sree Hari Rao, On random solutions of Volterra-Fredholm integral

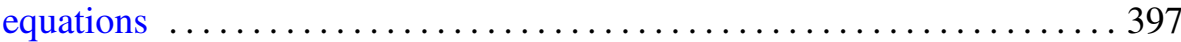

Takeyoshi Satō, Integral comparison theorems for relative Hardy spaces of solutions of the equations $\Delta u=P u$ on a Riemann surface $\ldots \ldots \ldots . .407$

Paul Sydney Selick, A reformulation of the Arf invariant one $\bmod p$ problem and applications to atomic spaces

Roelof Jacobus Stroeker, Reduction of elliptic curves over imaginary quadratic number fields

Jacob Towber, Natural transformations of tensor-products of representation-functors. I. Combinatorial preliminaries

James Chin-Sze Wong and Abdolhamid Riazi, Characterisations of amenable locally compact semigroups 